\title{
Distributed Measurement of Signal Power Evolution in a Phase Sensitive Parametric Amplifier
}

\author{
F. Alishahi ${ }^{1,3}$, A. Vedadi ${ }^{1}$, M. A. Soto ${ }^{2}$, A. Denisov ${ }^{2}$, K. Mehrany $^{3}$, L. Thévenaz ${ }^{2}$ and C-S Brès ${ }^{1}$ \\ 1. Photonic Systems Laboratory, Ecole Polytechnique Fédérale de Lausanne (EPFL) CH-1015 Lausanne, Switzerland \\ 2. Group for Fiber Optics, Ecole Polytechnique Fédérale de Lausanne (EPFL) CH-1015 Lausanne, Switzerland \\ 3. Electronics and Electrical Dept., Sharif University of Technology., Azadi Ave., Tehran, Iran \\ alishahi.fateme@gmail.com
}

\begin{abstract}
A method to measure the signal power evolution along phase-sensitive parametric amplifiers is proposed using Brillouin optical time-domain analysis. Different evolutions along the fiber are reported by varying input wave phases, including amplification and de-amplification.

OCIS codes: (060.2320) Fiber optics amplifiers and oscillators; 120.0280 Remote sensing and sensors
\end{abstract}

\section{Introduction}

The ever increasing demand for bandwidth and the introduction of multi-level coding of signal phase and amplitude in fiber optical communications systems has aroused a recent strong research interest in phase-sensitive fiber optical parametric amplifiers (PS-FOPAs). PS-FOPAs are based on the Kerr-induced four-wave mixing (FWM) between one or two pump wave(s), a signal and an idler wave along an optical fiber. The phase mismatch between pump(s), signal and idler dictates the efficiency and direction of the energy transfer between pump(s) and signal/idler. As a noticeable consequence, PS-FOPAs allow noise-free amplification of the in-phase signal and permit a drastic reduction of the noise figure in optical communications links, hence an increase in capacity [1]. Furthermore, phase sensitive amplification allows many ultrahigh speed processing functions of multi-level phase and amplitude coded signals including phase regeneration [2].

Similarly to their phase insensitive counterpart, the performance of PS-FOPAs may be impeded by longitudinal fluctuations of parameters such as dispersion [3]. Moreover, due to the sensitivity to the initial phase mismatch, a variety of combinations of amplification (i.e. pump transferring energy to signal/idler), and/or de-amplification (i.e signal/idler transferring energy back to the pump) can take place along the fiber leading to an overall gain/loss process. While many of these combinations could lead to a similar power levels at the fiber output, the noise figure might differ depending on the path of amplification/de-amplification that the signal has undergone. Measuring the different evolutions of the signal power along the PS-FOPA not only give an interesting insight in the phenomenon but can also be used to optimize the design of the amplifier.

In this work, distributed measurements of the signal power evolution along a PS-FOPA are performed for the first time to the best of our knowledge, using Brillouin optical time-domain analysis (BOTDA). The measurements depict different regimes of amplification, de-amplification or combination of both along the fiber.

\section{Operating principle and experimental setup}

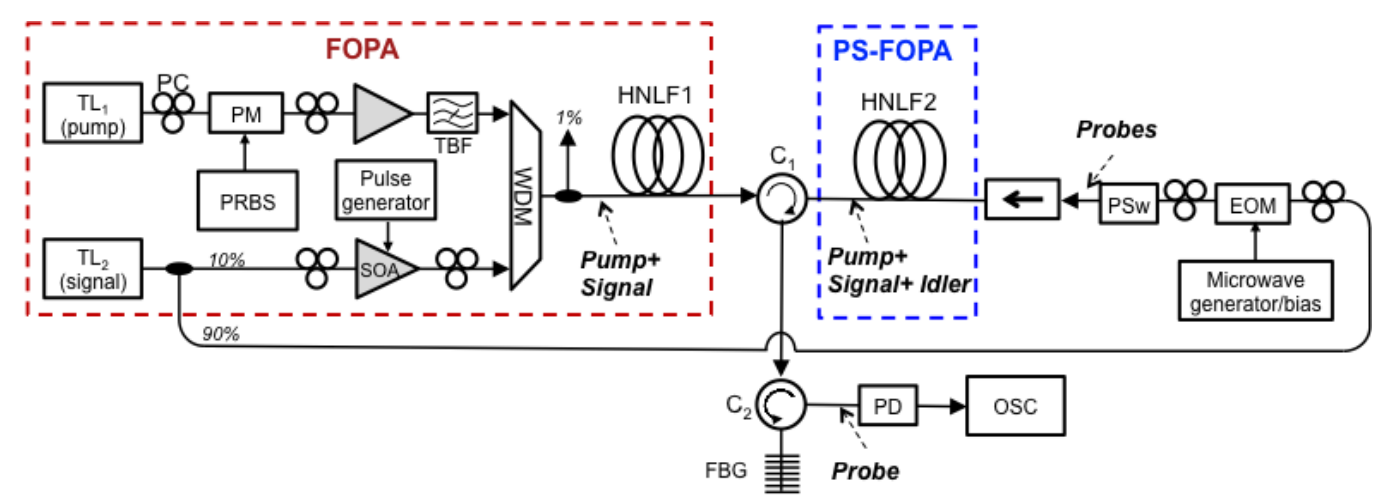

Fig 1: Experimental setup to perform distributed measurement along a PS-FOPA

The operating principle of the here proposed method relies on measuring the Brillouin interaction between a pulsed signal amplified along a PS-FOPA and a counter-propagating continuous-wave $(\mathrm{CW})$ probe spectrally shifted from 
the pulsed signal by the Brillouin frequency $v_{\mathrm{B}}$ in the fiber. When the probe interacts with the signal pulse, stimulated Brillouin scattering induces gain (loss) on the probe component located at a frequency $-v_{B}\left(+v_{B}\right)$ from the pulsed signal wavelength. The local Brillouin gain/loss, depending on the local signal energy, is measured monitoring the power variations of the probe in the time domain. Contrary to monitoring the evolution of a phase insensitive amplifier [4], both the signal and idler waves are launched together with the parametric pump into the fiber, thus leading to phase sensitive amplification. To practically set this condition, a phase insensitive FOPA has been used as a first stage for the initial generation of the idler wave in front of the BOTDA-probed PS-FOPA.

The experimental setup is depicted in Fig. 1. A parametric pump is generated using a $\mathrm{CW}_{\text {tunable }}$ laser $\left(\mathrm{TL}_{1}\right)$ followed by a phase modulator driven by a $10 \mathrm{GHz} 2^{17}-1$ long pseudo-random bit sequence in order to suppress stimulated Brillouin scattering (SBS) and an erbium-doped fiber amplifier (EDFA) to reach a peak power of $670 \mathrm{~mW}$ at the input of the first highly nonlinear fiber (HNLF1). A tunable $2 \mathrm{~nm}$ bandpass filter is used to filter out the amplified spontaneous emission (ASE) noise introduced by the EDFA in the pump. The signal and Brillouin probes are generated from a common CW tunable laser $\left(\mathrm{TL}_{2}\right)$ source. $10 \%$ of the $\mathrm{TL}_{2}$ power is directed into a gated semiconductor optical amplifier (SOA) for pulse generation. The pulsed signal and pump are coupled using a wavelength-division multiplexer (WDM) and sent through HNLF1. A 99/1 coupler is used at the HNLF1 input to monitor pump and signal powers. At the output of HNLF1, the pump, signal and generated idler are injected into the PS-FOPA through a circulator $\left(\mathrm{C}_{1}\right)$. The remaining $90 \%$ of $\mathrm{TL}_{2}$ is connected to a Mach-Zehnder modulator in order to generate a two-tone probe wave with high carrier suppression. To ensure that measured signal power evolution is related only to FWM along the PS-FOPA, and is not affected by potential Brillouin pump depletion, both sidebands are launched into the fiber [5]. A polarization switch (PSw) is used to sequentially acquire the probe traces along two orthogonal states of polarization and thus compensate fluctuations due to random birefringence along HNLF2. The probes are launched at the output of the PS-FOPA through an isolator. After counter propagation, the probes are directed to the remaining port of $\mathrm{C}_{1}$ and sent to a fiber Bragg grating (FBG) which selects either one of the probes before being acquired by the oscilloscope (OSC). Both HNLFs have their zero dispersion wavelength located at $1550 \mathrm{~nm}$ and are $500 \mathrm{~m}$ long each. The nonlinear coefficient is $11 \mathrm{~W}^{-1} \mathrm{~m}^{-1}$ for HNLF1 and $13 \mathrm{~W}^{-1} \mathrm{~m}^{-1}$ for HNLF2.

\section{Results and discussions}

The parametric pump wavelength is tuned to channels 32, 33 and 34 of the C-band ITU grid (1551.72 nm, $1550.92 \mathrm{~nm}$ and $1550.12 \mathrm{~nm}$ respectively). Using a CW signal at the input of HNLF1, the gain spectra are first measured after the phase insensitive FOPA. The amplification/de-amplification spectra of the PS-FOPA are then measured by comparing signal powers at the output and input of HNLF2, shown in Fig. 2. For each parametric pump, the spectrum exhibits oscillations up to $20 \mathrm{~dB}$ peak-to-peak, alternating between overall gain and loss. These oscillations are due to the strong dispersion induced by the SMF pigtails between HNLF1 output and HNLF2 input. The overall length of SMF is $5 \mathrm{~m}$ in this case, and allows for strong modification of the interacting waves phase mismatch [6] when changing either the signal wavelength and/or the pump wavelength.

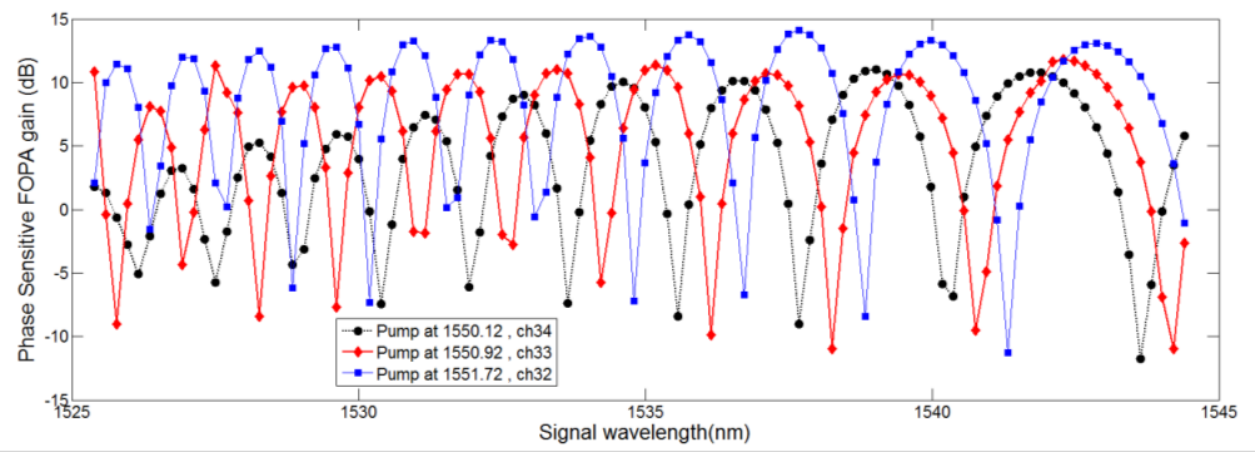

Fig. 1: PS-FOPA amplification/de-amplification spectrum for different pump wavelength positions.

To obtain the BOTDA traces the signal is tuned between 1527.8 and $1529 \mathrm{~nm}$. The peak power of the signal is comprised between $10 \mathrm{~mW}$ and $20 \mathrm{~mW}$ according to the signal position on the first stage gain spectrum. In order to eliminate the fluctuations resulting from variations in the Brillouin response along the fiber, standard pump-probe BOTDA measurements are first carried out in HNLF2 and subsequently used to normalize traces obtained in the PSFOPA. Fig. 3(a) depicts the evolution of signal gain/loss along the fiber as a function of the signal wavelength when the pump is located at $1550.12 \mathrm{~nm}$. Despite the varying behavior inside the fiber, the amplification/de-amplification typical oscillations are retrieved at the fiber output as expected; see Fig. 3(b). 
(a)

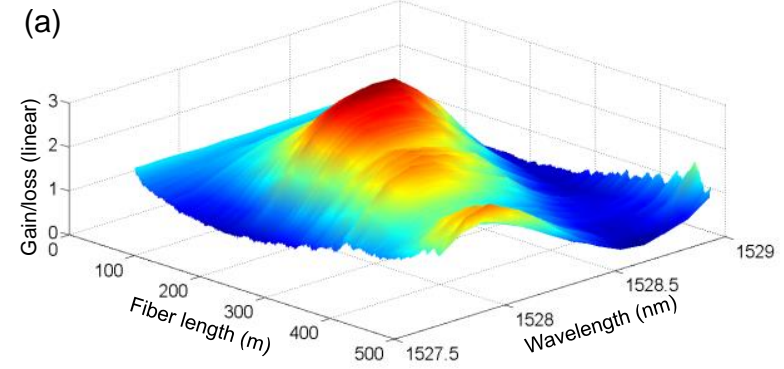

(b)

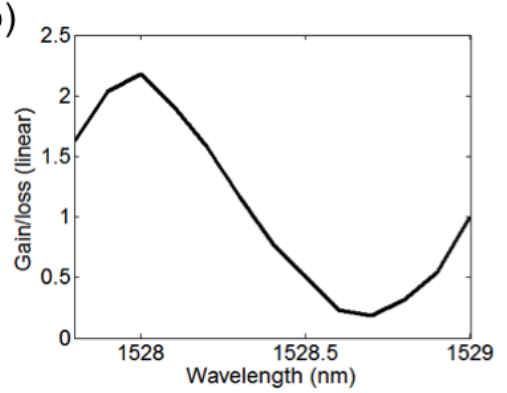

Fig. 2: (a) Signal amplification/de-amplification (linear unit) as a function of wavelength and location along the fiber; (b) Retrieved oscillation of gain/loss behavior from (a) at the end of the fiber

Fig. 4(a) shows the evolution of the signal gain/loss along the fiber for three different signal wavelengths. At $1528.3 \mathrm{~nm}$, the signal is continuously attenuated (red line in the figure). For a signal at $1528.1 \mathrm{~nm}$ (green line) or $1528.5 \mathrm{~nm}$ (blue line), the PS-FOPA operates close to transparency (i.e. the output gain is close to 1); however these signals evolve very differently along the PS-FOPA: the $1528.1 \mathrm{~nm}$ signal is first de-amplified and after amplified in the second half of the fiber, while the $1528.5 \mathrm{~nm}$ signal is first amplified and then de-amplified to reach transparency after $500 \mathrm{~m}$ of fiber. Finally, Fig 4(b) shows how different parametric gain/loss evolutions along the fiber can be obtained as a result of the different input phase mismatch when the pump wavelength is changed.

It is important to highlight that measuring the distributed evolution of the signal power along a PS-FOPA can have significant implications in the context of zero-dispersion wavelength (ZDW) mapping. Actually, the use of a phase-insensitive FOPA to map the distribution of the ZDW [5] suffers from two main drawbacks: $i$ ) the absence of idler at the beginning of the fiber induces uncertainties in the ZDW over the first meters of fiber, and ii) the risk of strong signal amplification could lead to parametric pump depletion. Using traces that oscillate close to transparency, as the one obtained with a PS-FOPA, can alleviate these two drawbacks and enable faster and more efficient ZDW retrieval.
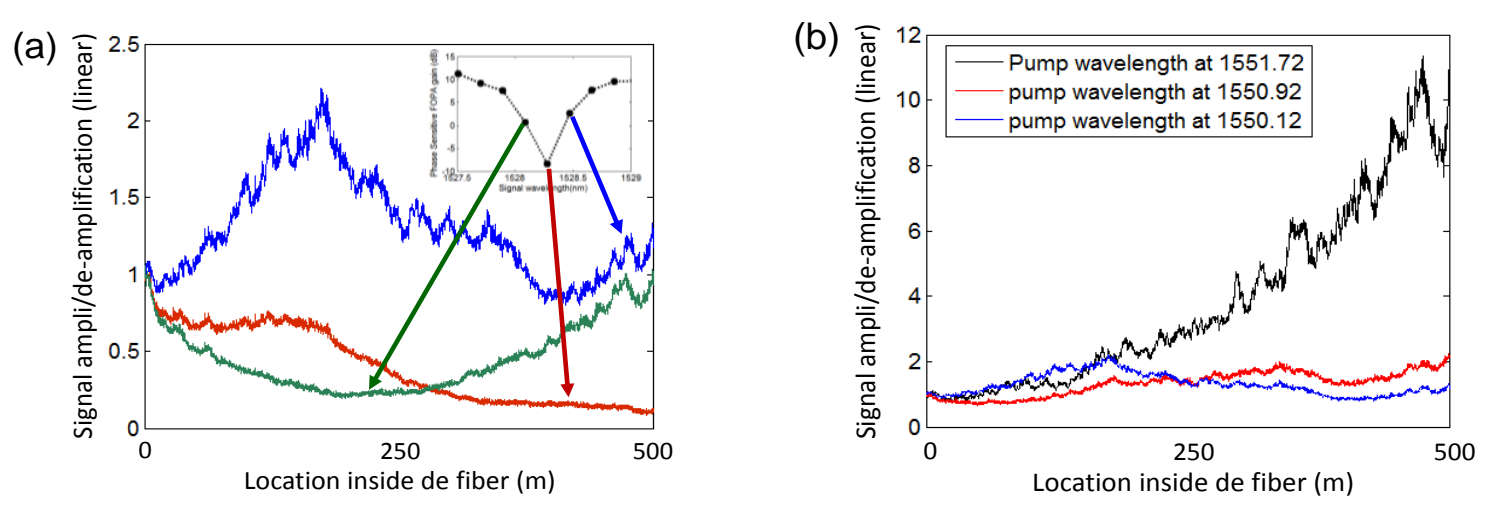

Fig. 3: Map of the signal distributtion for (a) fixed pump and different signal wavelengths and (b) fixed signal and different pump wavelengths.

In conclusion, a method to measure the distributed signal power evolution along a PS-FOPA has been proposed using Brillouin optical time-domain analysis. Local amplification and de-amplification of the signal were observed in various operating conditions. While the output value of the PS-FOPA might be identical at distinct signal wavelengths, the signal power may experience different possible amplification schemes as they propagate along the amplifier. Knowing the power evolution can be used to optimize the performance of amplifiers and could also be exploited for a more accurate ZDW mapping.

\section{References}

[1] Z. Tong et al., "Towards ultrasensitive optical links enabled by low-noise phase-sensitive amplifiers," Nature Photonics 5, 430-436 (2011).

[2] K. Croussore et al., "Phase-and-amplitude regeneration of differential phase-shift keyed signals using a phase-sensitive amplifier." Opt.

Express 14, (2006).

[3] F. Yaman et al., "Impact of dispersion fluctuations on dual-pump fiber-optic parametric amplifiers." IEEE Photon. Technol. Lett.16, (2004).

[4] A. Vedadi et al., "Brillouin optical time-domain analysis of fiber-optic parametric amplifiers." IEEE Photon. Technol. Lett.19, (2007).

[5] F. Alishahi et al., "Mapping Dispersion Fluctuations along Optical Fibers Using Brillouin Probing and a Fast Analytic Calculation," in CLEO:

2013, OSA Technical Digest (online) (Optical Society of America, 2013), paper CF1E.5.

[6] R. Tang et al., "Gain characteristics of a frequency non degenerate phase-sensitive fiber-optic parametric amplifier with phase self-stabilized input."Opt. Express 13, 10483-10493 (2005). 